\title{
Peristaltic Transport of a Jeffrey Fluid with Variable Viscosity through a Porous Medium in an Asymmetric Channel
}

\author{
A. Afsar Khan, ${ }^{1}$ R. Ellahi, ${ }^{1,2}$ and K. Vafai $^{2}$ \\ ${ }^{1}$ Department of Mathematics \& Statistics, FBAS, IIU, Islamabad, Pakistan \\ ${ }^{2}$ Department of Mechanical Engineering, University of California Riverside, USA \\ Correspondence should be addressed to R. Ellahi, rellahi@engr.ucr.edu
}

Received 12 December 2011; Accepted 16 February 2012

Academic Editor: Sanith Wijesinghe

Copyright (c) 2012 A. Afsar Khan et al. This is an open access article distributed under the Creative Commons Attribution License, which permits unrestricted use, distribution, and reproduction in any medium, provided the original work is properly cited.

The peristaltic flow of a Jeffrey fluid with variable viscosity through a porous medium in an asymmetric channel is investigated. The channel asymmetric is produced by choosing the peristaltic wave train on the wall of different amplitude and phase. The governing nonlinear partial differential equations for the Jeffrey fluid model are derived in Cartesian coordinates system. Analytic solutions for stream function, velocity, pressure gradient, and pressure rise are first developed by regular perturbation method, and then the role of pertinent parameters is illustrated graphically.

\section{Introduction}

Peristalsis is a mechanism to pump the fluid by means of moving contraction on the tubes or channel walls. This process has quite useful applications in many biological systems and industry. It occurs in swallowing food through the esophagus, chyme motion in the gastrointestinal tract, the vasomotion of small blood vessels such as venules, capillaries, and arterioles, urine transport from kidney to bladder, sanitary fluid transport of corrosive fluids, a toxic liquid transport in the nuclear industry, and so forth. In view of such physiological and industrial applications, the peristaltic flows has been studied with great interest by the various researchers for viscous and non-Newtonian fluids [1-9].

In most of the studies which deal with the peristaltic flows, the fluid viscosity is assumed to be constant. This assumption is not valid everywhere. In general the coefficients of viscosity for real fluids are functions of space coordinate, temperature, and pressure. For many liquids such as water, oils, and blood, the variation of viscosity due to space coordinate and temperature change is more dominant than other effects. Therefore, it is highly desirable 
to include the effect of variable viscosity instead of considering the viscosity of the fluid to be constant. Some important studies related to the variable viscosity are cited in [10-13].

A porous medium is the matter which contains a number of small holes distributed throughout the matter. Flows through a porous medium occur in filtration of fluids. Several investigations have been published by using generalized Darcy's law where the convective acceleration and viscous stress are taken into account [14-17].

Considering the importance of non-Newtonian fluid in peristalsis and keeping in mind the sensitivity of liquid viscosity, an attempt is made to study the peristaltic transport of Jeffrey having variable viscosity through a porous medium in a two-dimensional asymmetric channel under the assumption of long wave length and the low Reynolds number approximation. A regular perturbation method is used to solve the problem, and the solutions are expanded in a power series of viscosity parameter $\alpha$. The obtained expressions are utilized to discuss the influences of various emerging parameters.

\section{Mathematical Formulation}

We consider an incompressible Jeffrey fluid in an asymmetric channel of width $d_{1}+d_{2}$. A sinusoidal wave propagating with constant speed $c$ on the channel walls induces the flow. The wall surfaces are chosen of the following forms:

$$
\begin{gathered}
H_{1}(X, t)=a_{1}+b_{1} \cos \left[\frac{2 \pi}{\lambda}(X-c t)\right], \quad \text { upper wall, } \\
H_{2}(X, t)=-a_{2}-b_{2} \cos \left[\frac{2 \pi}{\lambda}(X-c t)+\phi\right], \quad \text { lower wall, }
\end{gathered}
$$

where $b_{1}, b_{2}$ are amplitude of the upper and lower waves, $\lambda$ is the wave length, $\phi$ is the phase difference which varies in the range $0 \leq \phi \leq \pi$. Furthermore, $a_{1}, a_{2}, b_{1}, b_{2}$, and $\phi$ should satisfy the following condition

$$
b_{1}^{2}+b_{2}^{2}+2 b_{1} b_{2} \cos \phi \leq\left(a_{1}+a_{2}\right)^{2}
$$

We assume that the flow becomes steady in the wave frame $(x, y)$ moving with velocity $c$ away from the fixed (laboratory) frame $(X, Y)$. The transformation between these two frames is given by

$$
x=X-c t, \quad y=Y, \quad u=U-c, \quad v=V, \quad p(x)=P(X, t),
$$


where $u$ and $v$ are the velocity components in the wave frame $(x, y), p$ and $P$ are pressure in wave and fixed frame of reference, respectively. The governing equations in the wave frame of reference are the Brinkman extended Daray equations given by

$$
\begin{gathered}
\frac{\partial u}{\partial x}+\frac{\partial v}{\partial y}=0 \\
\rho\left(u \frac{\partial u}{\partial x}+v \frac{\partial u}{\partial y}\right)=-\frac{\partial p}{\partial x}+\frac{1}{\varepsilon} \frac{\partial \tau_{x x}}{\partial x}+\frac{1}{\varepsilon} \frac{\partial \tau_{x y}}{\partial y}-\frac{\mu(y)}{k}(u+1) \\
\rho\left(u \frac{\partial v}{\partial x}+v \frac{\partial v}{\partial y}\right)=-\frac{\partial p}{\partial y}+\frac{\partial \tau_{y x}}{\partial x}+\frac{\partial \tau_{y y}}{\partial y}-\frac{\mu(y)}{k} v
\end{gathered}
$$

where

$$
\begin{aligned}
\tau_{x x} & =\frac{2 \mu(y)}{\left(1+\lambda_{1}\right)}\left[1+\lambda_{2}\left(u \frac{\partial}{\partial x}+v \frac{\partial}{\partial y}\right)\right] \frac{\partial u}{\partial x}, \\
\tau_{x y} & =\frac{\mu(y)}{\left(1+\lambda_{1}\right)}\left[1+\lambda_{2}\left(u \frac{\partial}{\partial x}+v \frac{\partial}{\partial y}\right)\right]\left(\frac{\partial u}{\partial y}+\frac{\partial v}{\partial x}\right), \\
\tau_{y y} & =\frac{2 \mu(y)}{\left(1+\lambda_{1}\right)}\left[1+\lambda_{2}\left(u \frac{\partial}{\partial x}+v \frac{\partial}{\partial y}\right)\right] \frac{\partial v}{\partial y},
\end{aligned}
$$

where $\lambda_{1}$ is the ratio of relaxation to retardation times, $\lambda_{2}$ is the retardation time, $\rho$ is the density, $k$ is the permeability of the porous medium, and $\varepsilon$ is the porosity of the porous medium.

Introducing the following nondimensional quantities:

$$
\begin{aligned}
& \bar{x}=\frac{x}{\lambda}, \quad \bar{y}=\frac{y}{a_{1}}, \quad \bar{u}=\frac{u}{c}, \quad \bar{v}=\frac{v}{c \delta}, \quad h_{1}=\frac{H_{1}}{a_{1}}, \quad h_{2}=\frac{H_{2}}{a_{1}}, \quad \bar{\tau}=\frac{a_{1} \tau}{\mu_{0} c} \\
& \bar{t}=\frac{c t}{\lambda}, \quad \mathrm{Da}=\frac{k}{a_{1}^{2}}, \quad \delta=\frac{a}{\lambda}, \quad \bar{p}=\frac{p a_{1}^{2}}{\mu_{0} c \lambda}, \quad a=\frac{b_{1}}{a_{1}}, \quad b=\frac{b_{2}}{a_{1}}, \quad d=\frac{a_{2}}{a_{1}} .
\end{aligned}
$$

With the help of (2.8), (2.4) to (2.6) after dropping the bars take the form

$$
\begin{gathered}
\frac{\partial u}{\partial x}+\frac{\partial v}{\partial y}=0 \\
\operatorname{Re} \delta\left(u \frac{\partial u}{\partial x}+v \frac{\partial u}{\partial y}\right)=-\frac{\partial p}{\partial x}+\frac{\delta}{\varepsilon} \frac{\partial \tau_{x x}}{\partial x}+\frac{1}{\varepsilon} \frac{\partial \tau_{x y}}{\partial y}-\frac{\mu(y)}{\mathrm{Da}}(u+1) \\
\operatorname{Re} \delta^{3}\left(u \frac{\partial v}{\partial x}+v \frac{\partial v}{\partial y}\right)=-\frac{\partial p}{\partial y}+\frac{\delta^{2}}{\varepsilon} \frac{\partial \tau_{x y}}{\partial x}+\frac{\delta}{\varepsilon} \frac{\partial \tau_{y y}}{\partial y}-\frac{\delta^{2} \mu(y)}{\mathrm{Da}} v
\end{gathered}
$$


where Darcy's number is

$$
\begin{gathered}
\mathrm{Da}=\frac{k}{a_{1}^{2}} \\
\tau_{x x}=\frac{2 \delta \mu(y)}{\left(1+\lambda_{1}\right)}\left[1+\frac{\lambda_{2} \delta c}{a_{1}}\left(u \frac{\partial}{\partial x}+v \frac{\partial}{\partial y}\right)\right] \frac{\partial u}{\partial x} \\
\tau_{x y}=\frac{\mu(y)}{\left(1+\lambda_{1}\right)}\left[1+\frac{\lambda_{2} \delta c}{a_{1}}\left(u \frac{\partial}{\partial x}+v \frac{\partial}{\partial y}\right)\right]\left(\frac{\partial u}{\partial y}+\delta^{2} \frac{\partial v}{\partial x}\right), \\
\tau_{y y}=\frac{2 \mu(y)}{\left(1+\lambda_{1}\right)}\left[1+\frac{\lambda_{2} \delta c}{a_{1}}\left(u \frac{\partial}{\partial x}+v \frac{\partial}{\partial y}\right)\right] \frac{\partial v}{\partial y} .
\end{gathered}
$$

Using the longwave length and small Reynolds number approximation, (2.10) and (2.11) take the form

$$
\begin{gathered}
\frac{\partial p}{\partial x}=\frac{1}{\varepsilon} \frac{\partial}{\partial y}\left[\frac{\mu(y)}{\left(1+\lambda_{1}\right)} \frac{\partial u}{\partial y}\right]-\frac{\mu(y)}{\mathrm{Da}}(u+1), \\
\frac{\partial p}{\partial y}=0
\end{gathered}
$$

The corresponding boundary conditions are

$$
\begin{aligned}
& u=-1, \quad \text { at } y=h_{1}, \\
& u=-1, \quad \text { at } y=h_{2},
\end{aligned}
$$

where

$$
h_{1}=1+a \cos 2 \pi x, \quad h_{2}=-d-b \cos (2 \pi x+\phi) .
$$

Equation (2.14) indicate that $p$ is independent of $y$. Therefore, (2.10) can be written as

$$
\frac{d p}{d x}=\frac{1}{\varepsilon} \frac{\partial}{\partial y}\left[\frac{\mu(y)}{\left(1+\lambda_{1}\right)} \frac{\partial u}{\partial y}\right]-\frac{\mu(y)}{\mathrm{Da}}(u+1),
$$

where $\mu(y)$ is the viscosity variation on peristaltic flow. For the present analysis, we assume viscosity variation in the dimensionless form [10]:

$$
u(y)=e^{-\alpha y}, \quad u(y)=1-\alpha y+\frac{\alpha y^{2}}{2}, \quad \text { for } \alpha \prec \prec 1
$$


The volume flow rate in the wave frame is given by

$$
q=\int_{h_{2}}^{h_{1}} u d y
$$

The instantaneous flux $Q(x, t)$ in the laboratory frame is defined as

$$
Q(x, t)=\int_{h_{1}}^{h_{2}}(u+1) d y=q+h_{1}-h_{2} .
$$

The average flux over one period $(T=\mathcal{l} / c)$ is given by

$$
\bar{Q}=\frac{1}{T} \int_{0}^{T} Q d t=\frac{1}{T} \int_{0}^{T}\left(q+h_{1}-h_{2}\right) d t=q+1+d
$$

\section{Perturbation Solution}

Equation (2.16) is a nonlinear differential equation so that it is not possible to obtain a closed form solution; so we seek perturbation solution. We expand $u, p$ and $q$ as

$$
\begin{aligned}
& u=u_{0}+\alpha u_{1}+\alpha^{2} u_{2}+o\left(\alpha^{3}\right) \\
& p=p_{0}+\alpha p_{1}+\alpha^{2} p_{2}+o\left(\alpha^{3}\right) \\
& q=q_{0}+\alpha q_{1}+\alpha^{2} q_{2}+o\left(\alpha^{3}\right) .
\end{aligned}
$$

Substituting these equations into (2.15a), (2.15b), (2.15c), and (2.16), we have the following system of equations.

\subsection{Zeroth-Order Equations $\alpha^{0}$}

$$
\frac{\partial^{2} u_{0}}{\partial y^{2}}-N^{2} u_{0}=\varepsilon\left(1+\lambda_{1}\right) \frac{d p_{0}}{d x}+N^{2}
$$

where

$$
\begin{gathered}
N=\frac{\varepsilon\left(1+\lambda_{1}\right)}{\mathrm{Da}}, \\
u_{0}=-1, \quad \text { at } y=h_{1}, h_{2} .
\end{gathered}
$$




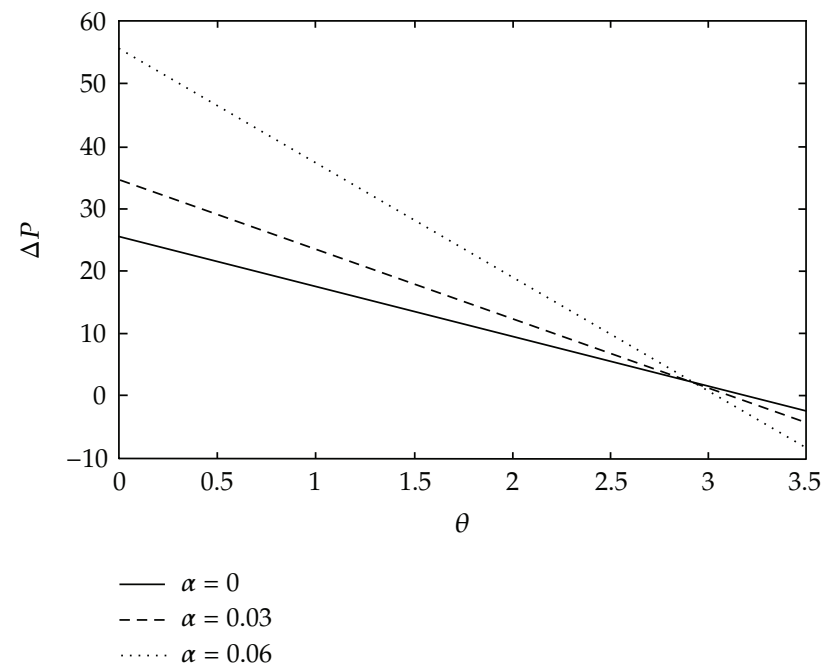

Figure 1: The pressure rise versus flow rate when $a=0.2, b=0.6, d=0.8, \varepsilon=0.3, \lambda_{1}=0.8, \mathrm{Da}=0.6$, and $\phi=\pi / 4$.

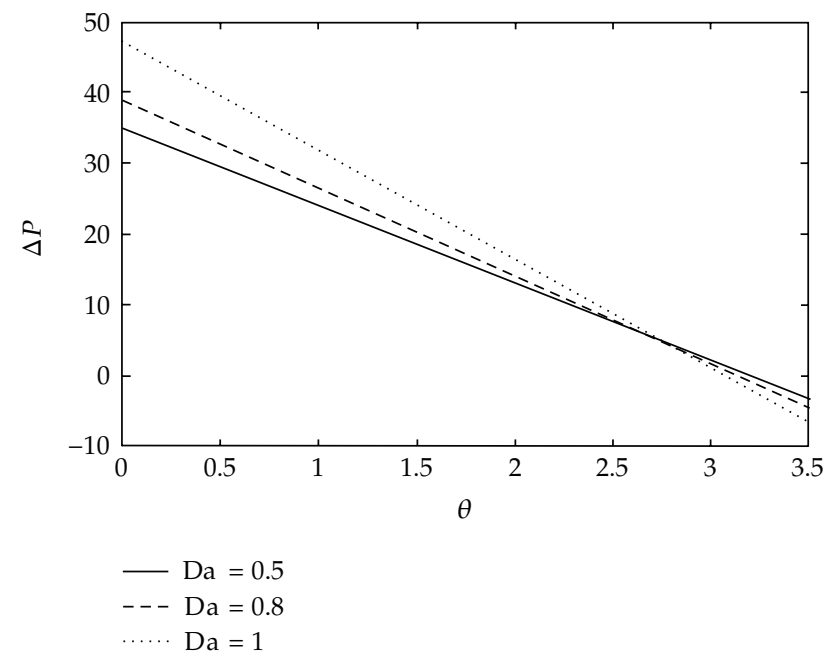

Figure 2: The pressure rise verses flow rate when $\alpha=0.01, a=0.2, b=0.6, d=0.8, \varepsilon=0.3, \lambda_{1}=0.4$, and $\phi=\pi / 4$.

\subsection{First-Order Equations $\alpha$}

$$
\begin{gathered}
\frac{\partial^{2} u_{1}}{\partial y^{2}}-N^{2} u_{1}=\varepsilon\left(1+\lambda_{1}\right) \frac{d p_{1}}{d x}+\varepsilon y\left(1+\lambda_{1}\right) \frac{d p_{0}}{d x}+\frac{\partial u_{0}}{\partial y}, \\
u_{1}=0, \quad \text { at } y=h_{1}, h_{2} .
\end{gathered}
$$




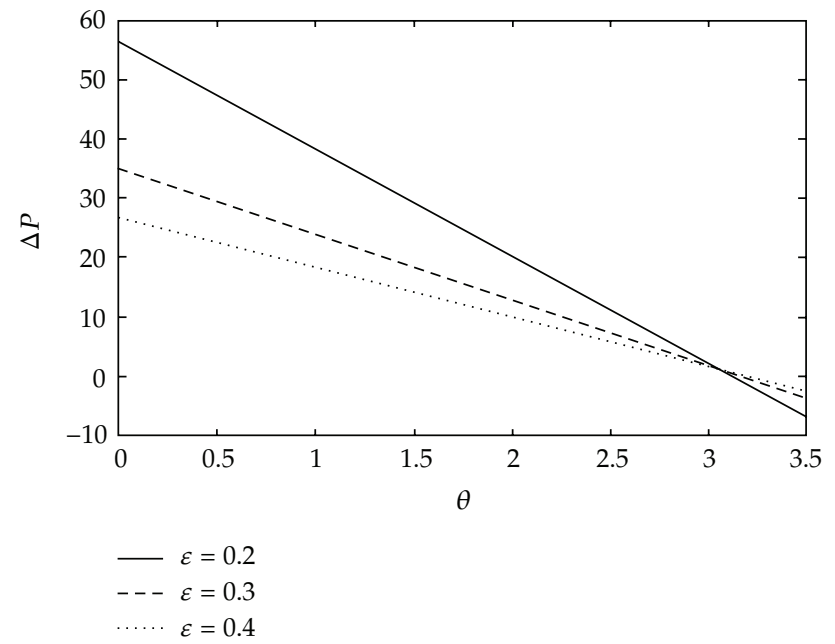

Figure 3: The pressure rise verses flow rate when $\alpha=0.01, a=0.2, b=0.6, d=0.8, \lambda_{1}=0.4, \mathrm{Da}=0.5$, and $\phi=\pi / 4$.

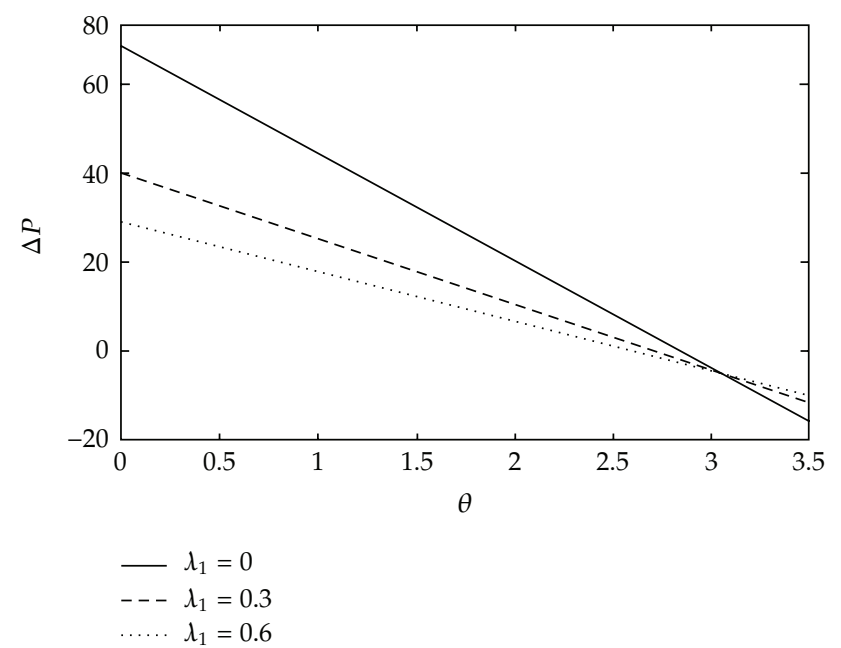

Figure 4: The pressure rise verses flow rate when $\alpha=0.01, a=0.2, b=0.6, d=0.8, \varepsilon=0.3, \mathrm{Da}=0.8$, and $\phi=\pi / 4$.

\subsection{Second-Order Equations $\alpha^{2}$}

$$
\begin{gathered}
\frac{\partial^{2} u_{2}}{\partial y^{2}}-N^{2} u_{2}=\varepsilon\left(1+\lambda_{1}\right) \frac{d p_{2}}{d x}+\varepsilon\left(1+\lambda_{1}\right) y \frac{d p_{1}}{d x}+\frac{y^{2}}{2} \varepsilon\left(1+\lambda_{1}\right) \frac{d p_{0}}{d x}+\frac{\partial u_{1}}{\partial y} \\
u_{2}=0 \quad \text { at } y=h_{1}, h_{2} .
\end{gathered}
$$




\subsection{Zeroth-Order Solution}

Solving (3.2) and (3.3), we get

$$
u_{0}=\frac{\varepsilon\left(1+\lambda_{1}\right)}{N^{2}} \frac{d p_{0}}{d x}\left[C_{1} \cosh N y+C_{2} \sinh N y-1\right]-1,
$$

where

$$
C_{1}=\frac{\left(\sinh N h_{1}-\sinh N h_{2}\right)}{\sinh N\left(h_{1}-h_{2}\right)}, \quad C_{2}=\frac{\left(\cosh N h_{2}-\cosh N h_{1}\right)}{\sinh N\left(h_{1}-h_{2}\right)}
$$

and the volume flow rate $q_{0}$ is given by

$$
q_{0}=\int_{h_{2}}^{h_{1}} u_{0} d y
$$

From (3.8), we have

$$
\frac{d p_{0}}{d x}=\left(q_{0}+h_{1}-h_{2}\right) A
$$

where

$$
A=\frac{N^{3} \sinh N\left(h_{1}-h_{2}\right)}{\varepsilon\left(1+\lambda_{1}\right)\left[2 \cosh N\left(h_{1}-h_{2}\right)-2-\left(h_{1}-h_{2}\right) N \sinh N\left(h_{1}-h_{2}\right)\right]} .
$$

The dimensionless pressure rise at this order is

$$
\Delta P_{0}=\int_{0}^{1} \frac{d p_{0}}{d x} d x
$$

\subsection{First-Order Solution}

Substituting zeroth order solution (3.8) into (3.4) and then solving the resulting system along with the corresponding boundary conditions, we arrive at

$$
\begin{aligned}
u_{1}=\frac{\varepsilon\left(1+\lambda_{1}\right)}{N^{2}} \frac{d p_{1}}{d x}[ & \left.C_{1} \cosh N y+C_{2} \sinh N y-1\right] \\
+\frac{\varepsilon\left(1+\lambda_{1}\right)}{2 N^{2}} \frac{d p_{0}}{d x}[ & -2 y+C_{1} y \cosh N y+C_{2} y \sinh N y \\
& \times \frac{\sinh N y\left(h_{1} \cosh N h_{2}-h_{2} \cosh N h_{1}\right)}{\sinh N\left(h_{1}-h_{2}\right)} \\
& \left.+\frac{\cosh N y\left(h_{2} \sinh N h_{1}-h_{1} \sinh N h_{2}\right)}{\sinh N\left(h_{1}-h_{2}\right)}\right],
\end{aligned}
$$




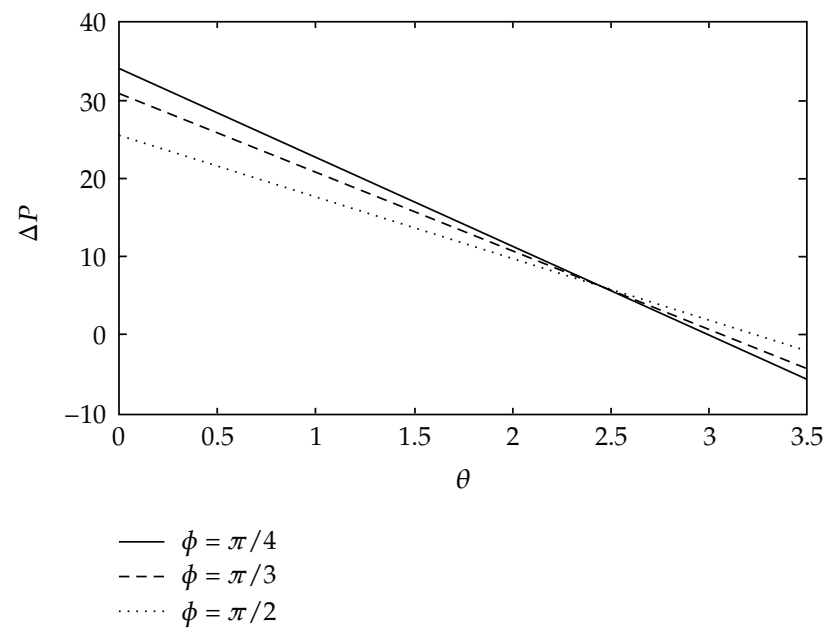

Figure 5: The pressure rise verses flow rate when $\alpha=0.01, a=0.4, b=0.6, d=0.8, \varepsilon=0.4, \lambda_{1}=0.5$, and $\mathrm{Da}=0.5$.

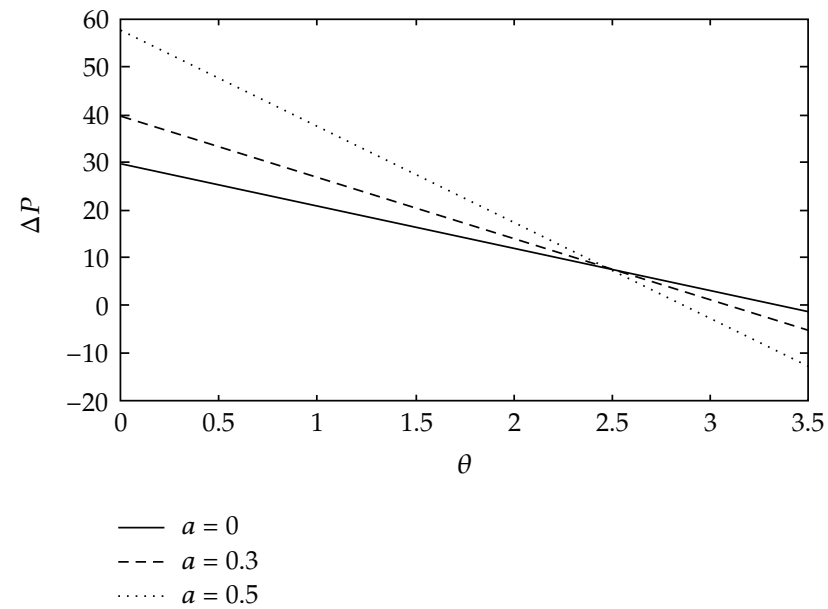

Figure 6: The pressure rise verses flow rate when $\alpha=0.01, b=0.6, d=0.8, \varepsilon=0.3, \lambda_{1}=0.4$, Da $=0.5$, and $\phi=\pi / 4$.

and the volume flow rate $q_{1}$ is given by

$$
q_{1}=\int_{h_{2}}^{h_{1}} u_{1} d y
$$

From (3.14), we get

$$
\frac{d p_{1}}{d x}=A q_{1}+\frac{A \varepsilon\left(1+\lambda_{1}\right)}{2 N^{3}} \frac{d p_{0}}{d x}\left[N^{2}\left(h_{1}^{2}-h_{2}^{2}\right)+\frac{\left(h_{1}+h_{2}\right)\left(1-\cosh N\left(h_{1}-h_{2}\right)\right)}{\sinh N\left(h_{1}-h_{2}\right)}\right] .
$$




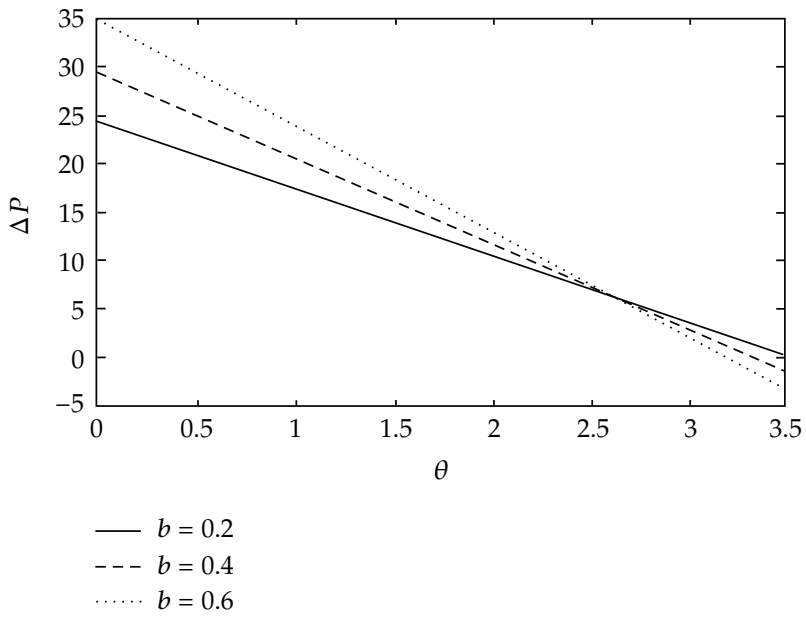

Figure 7: The pressure rise verses flow rate when $\alpha=0.01, a=0.2, d=0.8, \varepsilon=0.3, \lambda_{1}=0.4, \mathrm{Da}=0.5$, and $\phi=\pi / 4$.

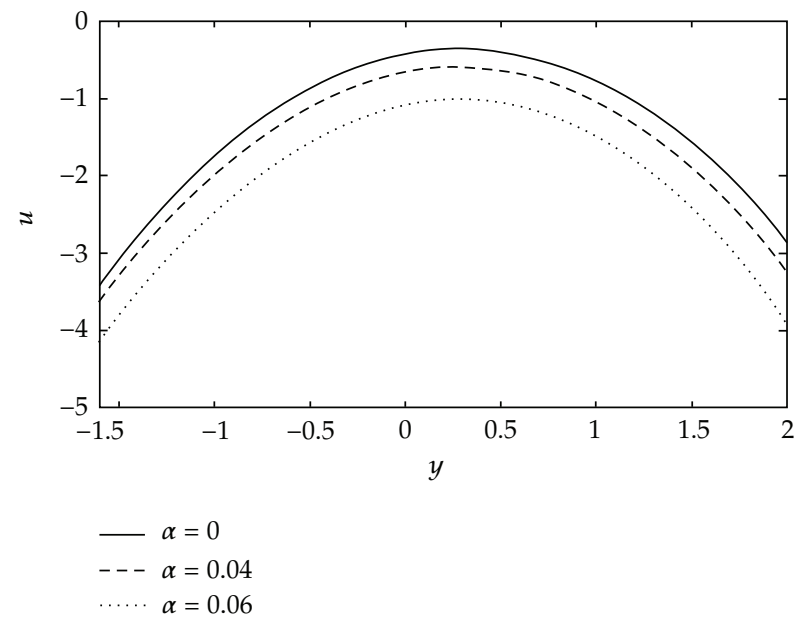

Figure 8: Axial velocity versus $y$ at $a=0.2, b=0.6, d=0.8, \varepsilon=0.2, \lambda_{1}=1, \mathrm{Da}=1, x=\pi / 6, q=-1$, and $\phi=\pi / 2$.

The dimensionless pressure rise at this order is

$$
\Delta P_{1}=\int_{0}^{1} \frac{d p_{1}}{d x} d x
$$




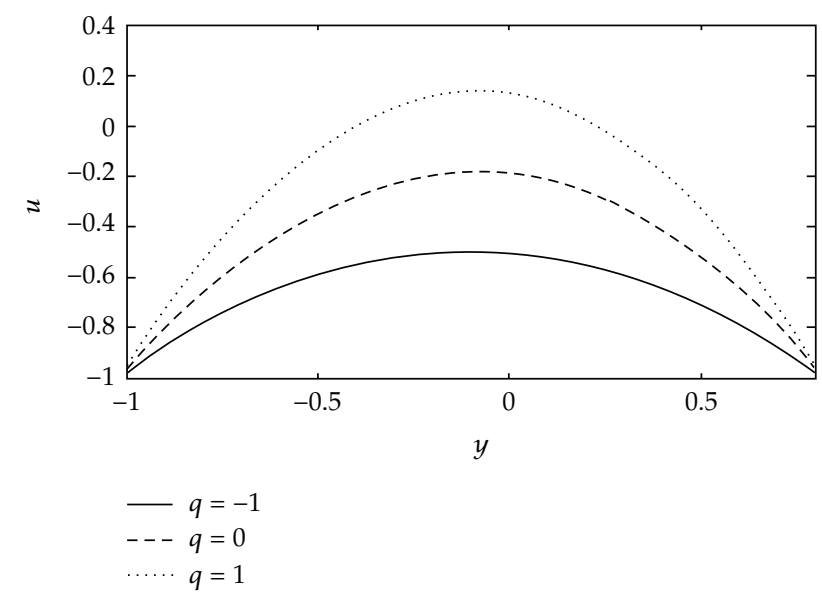

Figure 9: Axial velocity versus $y$ at $\alpha=0.05, a=0.2, b=0.6, d=0.8, \varepsilon=0.2, \lambda_{1}=1, \mathrm{Da}=1, x=0$, and $\phi=\pi / 2$.

\subsection{Second-Order Solution}

Solving (3.6) by using (3.8) and (3.14) and the boundary condition (3.5), we obtain

$$
\begin{aligned}
u_{2}=\frac{\varepsilon\left(1+\lambda_{1}\right)}{N^{2}} \frac{d p_{2}}{d x}[ & \left.C_{1} \cosh N y+C_{2} \sinh N y-1\right] \\
+\frac{\varepsilon\left(1+\lambda_{1}\right)}{2 N^{2}} \frac{d p_{1}}{d x}[ & \frac{\sinh N y\left(h_{1} \cosh N h_{2}-h_{2} \cosh N h_{1}\right)}{\sinh N\left(h_{1}-h_{2}\right)}-2 y \\
& +C_{1} y \cosh N y+C_{2} y \sinh N y \\
& \left.+\frac{\cosh N y\left(h_{2} \sinh N h_{1}-h_{1} \sinh N h_{2}\right)}{\sinh N\left(h_{1}-h_{2}\right)}\right] \\
+\frac{d p_{0}}{d x} \frac{\varepsilon\left(1+\lambda_{1}\right)}{4 N^{2}}\left[\frac{C_{1}\left(y \sinh N y+N y^{2} \cosh N y\right)+C_{2}\left(y \cosh N y+y^{2} N \sinh N y\right)}{2 N}\right. & -y^{2}+\frac{\left(1-\cosh N\left(h_{1}-h_{2}\right)\right)\left(\sinh N y\left(h_{1} \cosh N h_{2}+h_{2} \cosh N h_{1}\right)\right)}{2 \sinh N\left(h_{1}-h_{2}\right)} \\
& -\frac{\left(1-\cosh N\left(h_{1}-h_{2}\right)\right)\left(\left(h_{1} \sinh N h_{2}+h_{2} \sinh N h_{1}\right) \cosh N y\right)}{2 \sinh h^{2} N\left(h_{1}-h_{2}\right)} \\
& -\frac{\left(h_{1} \cosh N h_{2}-h_{2} \cosh N h_{1}\right)\left(C_{1} y \cosh N y+C_{2} y \sinh N y\right)}{\left(\cosh N h_{1}-\cosh N h_{2}\right)} \\
& +\frac{\left(h_{2}^{2} \cosh N h_{1}-h_{1}^{2} \cosh N h_{2}\right) \sinh N y}{2 \sinh N\left(h_{1}-h_{2}\right)}
\end{aligned}
$$




$$
\begin{aligned}
& +\frac{\left(h_{1}^{2} \sinh N h_{1}-h_{2}^{2} \sinh N h_{2}\right) \cosh N y}{2 \sinh N\left(h_{1}-h_{2}\right)} \\
& \left.\times \frac{8\left(1-C_{1} \cosh N y-C_{2} \sinh N y\right)}{N^{2}}+\frac{\left(h_{1}-h_{2}\right) y \cosh N y}{\left(\cosh N h_{1}-\cosh N h_{2}\right)}\right],
\end{aligned}
$$

and the volume flow rate $q_{2}$ is given by

$$
q_{2}=\int_{h_{2}}^{h_{1}} u_{2} d y
$$

From (3.18), we have

$$
\begin{aligned}
\frac{d p_{2}}{d x}= & A q_{2}+\frac{A \varepsilon\left(1+\lambda_{1}\right)}{2 N^{3}} \frac{d p_{1}}{d x}\left[\frac{2\left(h_{1}+h_{2}\right)\left(1-\cosh N\left(h_{1}-h_{2}\right)\right)}{\sinh N\left(h_{1}-h_{2}\right)}+N\left(\left(h_{1}^{2}-h_{2}^{2}\right)\right)\right] \\
& -\frac{A \varepsilon\left(1+\lambda_{1}\right)}{4 N^{3}} \frac{d p_{0}}{d x}\left[\frac{8\left(h_{1}-h_{2}\right)}{N}-\frac{3\left(h_{1}-h_{2}\right)}{2 N}-\left(\frac{h_{1}^{3}}{3}-\frac{h_{2}^{3}}{3}\right)\right] \\
& +\frac{\left(h_{1}^{2}+h_{2}^{2}\right)\left(1-\cosh N\left(h_{1}-h_{2}\right)\right)}{2 N \sinh N\left(h_{1}-h_{2}\right)}+\frac{\left(h_{1}-h_{2}\right)\left(h_{1} \sin N h_{1}-h_{2} \sin N h_{2}\right)}{\left(\cosh N h_{1}-\cosh N h_{2}\right)} \\
& +\frac{\left(h_{1}+h_{2}\right)\left(1-\cosh N\left(h_{1}-h_{2}\right)\right)\left(h_{1} \cosh N h_{2}-h_{2} \cosh N h_{1}\right)}{\sinh N\left(h_{1}-h_{2}\right)\left(\cosh N h_{1}-\cosh N h_{2}\right)} \\
& +\frac{\left(h_{1}^{2} N^{2}+h_{2}^{2} N^{2}+2\right)\left(\cosh N\left(h_{1}-h_{2}\right)-1\right)}{2 N^{2} \sinh N\left(h_{1}-h_{2}\right)}+\frac{16\left(1-\cosh N\left(h_{1}-h_{2}\right)\right)}{N^{2} \sinh N\left(h_{1}-h_{2}\right)} .
\end{aligned}
$$

The dimensionless pressure rise at this order is

$$
\Delta P_{2}=\int_{0}^{2} \frac{d p_{2}}{d x} d x
$$

Summarizing the result obtained from (3.11), (3.16), and (3.20), we write

$$
\Delta P=\Delta P_{0}+\alpha \Delta P_{1}+\alpha^{2} \Delta P_{2} .
$$

Corresponding stream functions can be defined as

$$
u=\frac{\partial \Psi}{\partial y}, \quad v=-\delta \frac{\partial \Psi}{\partial x}
$$




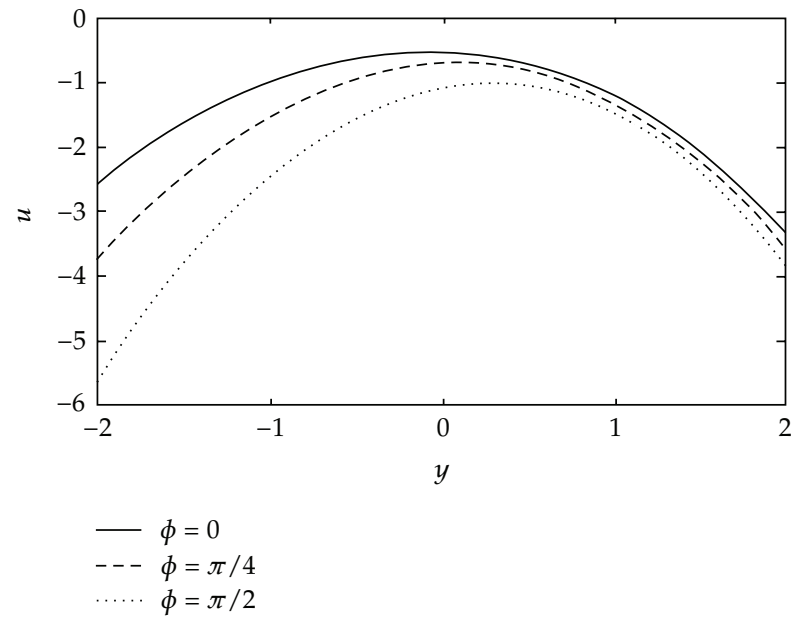

Figure 10: Axial velocity versus $y$ at $\alpha=0.05, a=0.2, b=0.6, d=0.8, \varepsilon=0.2, \lambda_{1}=1, \mathrm{Da}=1, x=\pi / 2$, and $q=-1$.

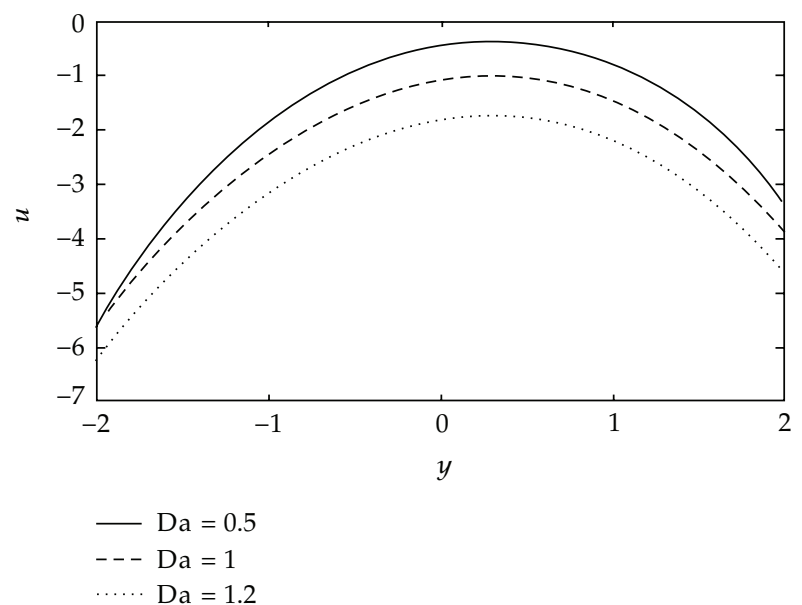

Figure 11: Axial velocity versus $y$ at $\alpha=0.05, a=0.2, b=0.6, d=0.8, \varepsilon=0.2, \lambda_{1}=1, q=-1, x=\pi / 6$, and $\phi=\pi / 2$.

\section{Results and Discussion}

We have used a regular perturbation series in term of the dimensional viscosity parameter $\alpha$ to obtain analytical solution of the field equations for peristaltic flow of Jeffrey fluid in an asymmetric channel. To study the behavior of solutions, numerical calculations for several values of viscosity parameter $\alpha$, Daray number Da, porosity $\varepsilon$, amplitude ratio $\phi$, Jeffrey fluid parameter $\lambda_{1}, a$ and $b$ have been calculated numerically using MATHEMATICA software.

Figure 1 shows the variation of $\Delta P$ with flow rate $\theta$ for different values of $\alpha$. It is depicted that the time-average flux $\theta$ increase with increasing the viscosity parameter $\alpha$. Figure 2 represents the variation of $\Delta P$ with the flow rate $\theta$ for different values of Da. We observe that an increase in the peristaltic pumping rate pressure rises. Figures 3 and 4 are graphs of pressure rise $\Delta P$ with the flow rate $\theta$ for values of $\varepsilon$ and $\lambda_{1}$. It is observed that the 


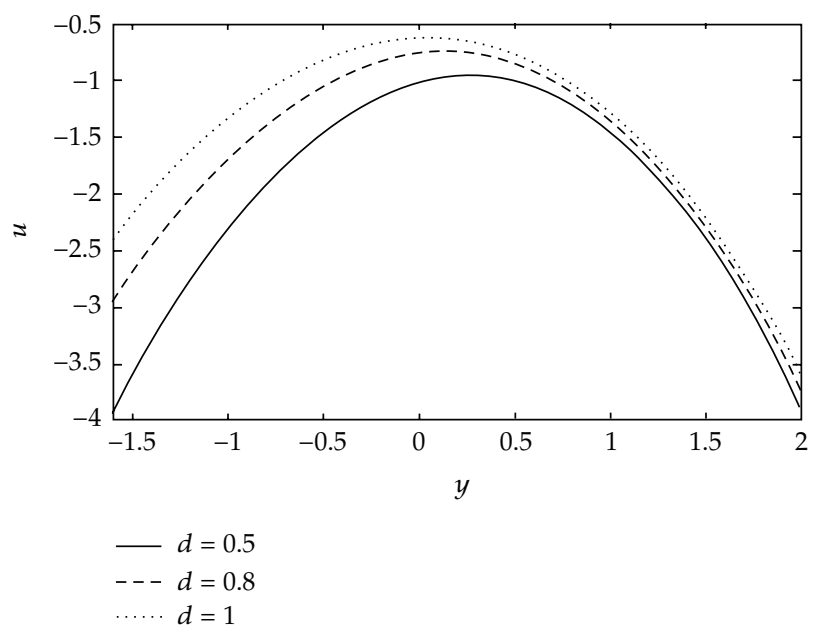

Figure 12: Axial velocity versus $y$ at $\alpha=0.05, a=0.2, b=0.6, q=-1, \varepsilon=0.2, \lambda_{1}=1, \mathrm{Da}=1, x=\pi / 6$, and $\phi=\pi / 2$.

pumping rate decreases with increase of $\varepsilon$ and $\lambda_{1}$. Figure 5 is the graph of the variation of $\Delta P$ versus the flow rate $\theta$ for different values of phase difference $\phi$. It is observed that the pumping rate decreases with the increase of $\phi$. Figures 6 and 7 plot the relation between pressure rise $\Delta P$ and flow rate $\theta$ for different values of $a$ and $b$, respectively. Figure 8 represents the graph of axial velocity $u$ versus $y$. It can be seen that an increase in $\alpha$ decreases the magnitude of axial velocity $u$. The effects of $q$ on the axial velocity $u$ are seen through Figure 9. It is noticed that an increase in $q$ increase the magnitude of the axial velocity. Figures 10 and 11 illustrate the effect of phase difference $\phi$ and Daray's number Da on the axial velocity $u$. It is observed that the magnitude of axial velocity decreases with the increasing phase difference $\phi$ and Daray's number Da. In Figure 12 the axial velocity $u$ is graphed versus $y$. We note that the magnitude of axial velocity increases as the channel width $d$ increases. It is worth mentioning that in the absence of porosity parameter the solutions of [10] can be derived as special case of the present analysis. This provides the useful check. It may be remarked that the problem for this particular model was not solved earlier even by any traditional perturbation technique. The results presented in this paper will now be available for experimental verification.

\section{Acknowledgments}

R. Ellahi thanks to United State Education Foundation Pakistan and CIES USA for honoring him by the Fulbright Scholar Award for the year 2011-2012. R. Ellahi also grateful to the Higher Education Commission and PCST of Pakistan for awarding him with the awards of NRPU and Productive Scientist, respectively.

\section{References}

[1] M. Y. Malik, A. Hussain, and S. Nadeem, "Flow of a Jeffrey-six constant fluid between coaxial cylinders with heat transfer," Communications in Theoretical Physics, vol. 56, pp. 345-351, 2011. 
[2] W. Tan and T. Masuoka, "Stokes' first problem for a second grade fluid in a porous half-space with heated boundary," International Journal of Non-Linear Mechanics, vol. 40, no. 4, pp. 515-522, 2005.

[3] M. Hameed and S. Nadeem, "Unsteady MHD flow of a non-Newtonian fluid on a porous plate," Journal of Mathematical Analysis and Applications, vol. 325, no. 1, pp. 724-733, 2007.

[4] F. M. Mahomed and T. Hayat, "Note on an exact solution for the pipe flow of a third-grade fluid," Acta Mechanica, vol. 190, no. 1-4, pp. 233-236, 2007.

[5] K. S. Mekheimer and Y. Abd elmaboud, "The influence of heat transfer and magnetic field on peristaltic transport of a Newtonian fluid in a vertical annulus: application of an endoscope," Physics Letters A, vol. 372, no. 10, pp. 1657-1665, 2008.

[6] K. S. Mekheimer, "Effect of the induced magnetic field on peristaltic flow of a couple stress fluid," Physics Letters A, vol. 372, no. 23, pp. 4271-4278, 2008.

[7] S. Srinivas and M. Kothandapani, "Peristaltic transport in an asymmetric channel with heat transfera note," International Communications in Heat and Mass Transfer, vol. 35, no. 4, pp. 514-522, 2008.

[8] M. Kothandapani and S. Srinivas, "Peristaltic transport of a Jeffrey fluid under the effect of magnetic field in an asymmetric channel," International Journal of Non-Linear Mechanics, vol. 43, no. 9, pp. 915924, 2008.

[9] M. Dehghan and F. Shakeri, "The numerical solution of the second painlevé equation," Numerical Methods for Partial Differential Equations, vol. 25, no. 5, pp. 1238-1259, 2009.

[10] S. Nadeem and N. S. Akbar, "Peristaltic flow of a Jeffrey fluid with variable viscosity in an asymmetric channel," Zeitschrift fur Naturforschung A , vol. 64, no. 11, pp. 713-722, 2009.

[11] S. Nadeem and N. S. Akbar, "Effects of heat transfer on the peristaltic transport of MHD Newtonian fluid with variable viscosity: application of Adomian decomposition method," Communications in Nonlinear Science and Numerical Simulation, vol. 14, no. 11, pp. 3844-3855, 2009.

[12] A. Ebaid, "A new numerical solution for the MHD peristaltic flow of a bio-fluid with variable viscosity in a circular cylindrical tube via Adomian decomposition method," Physics Letters A, vol. 372, no. 32, pp. 5321-5328, 2008.

[13] A. El Hakeem, A. El Naby, A. E. M. El Misiery, and I. I. El Shamy, "Hydromagnetic flow of fluid with variable viscosity in a uniform tube with peristalsis," Journal of Physics A, vol. 36, no. 31, pp. 8535-8547, 2003.

[14] S. Srinivas and R. Gayathri, "Peristaltic transport of a Newtonian fluid in a vertical asymmetric channel with heat transfer and porous medium," Applied Mathematics and Computation, vol. 215, no. 1, pp. 185-196, 2009.

[15] S. Srinivas and M. Kothandapani, "The influence of heat and mass transfer on MHD peristaltic flow through a porous space with compliant walls," Applied Mathematics and Computation, vol. 213, no. 1, pp. 197-208, 2009.

[16] S. Nadeem, N. S. Akbar, and M. Y. Malik, "Numerical solutions of peristaltic flow of a newtonian fluid under the effects ofmagnetic field and heat transfer in a porous concentric tubes," Zeitschrift fur Naturforschung A, vol. 65, no. 5, pp. 369-380, 2010.

[17] E. F. Elshehawey, N. T. Eldabe, E. M. Elghazy, and A. Ebaid, "Peristaltic transport in an asymmetric channel through a porous medium," Applied Mathematics and Computation, vol. 182, no. 1, pp. 140-150, 2006. 


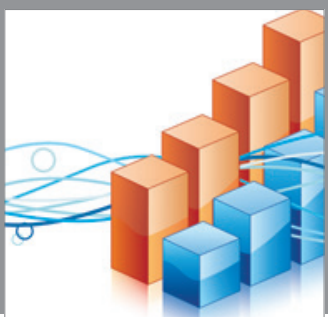

Advances in

Operations Research

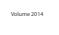

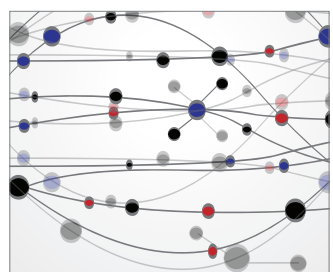

\section{The Scientific} World Journal
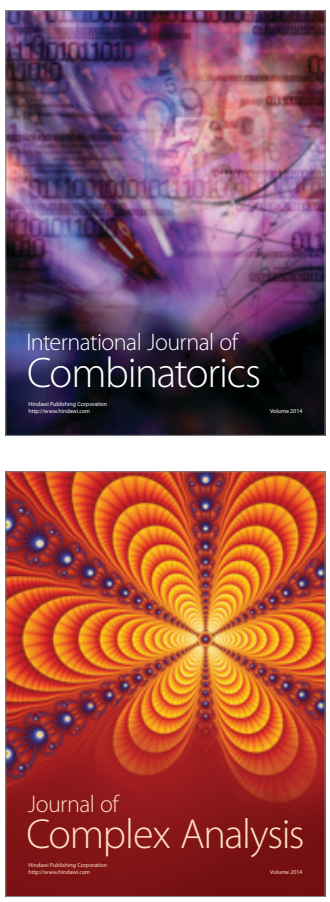

International Journal of

Mathematics and

Mathematical

Sciences
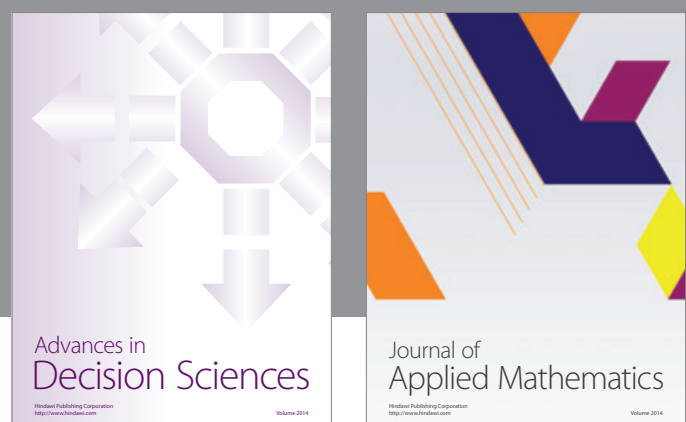

Journal of

Applied Mathematics
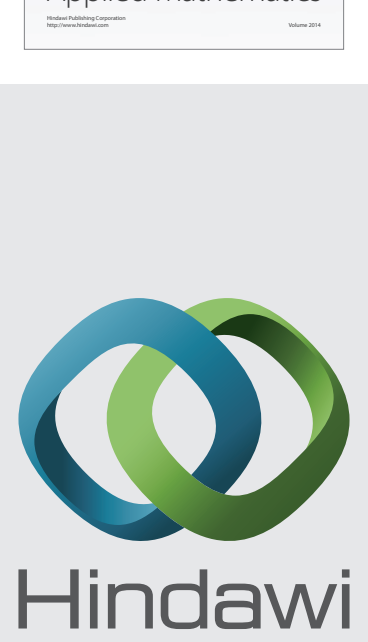

Submit your manuscripts at http://www.hindawi.com
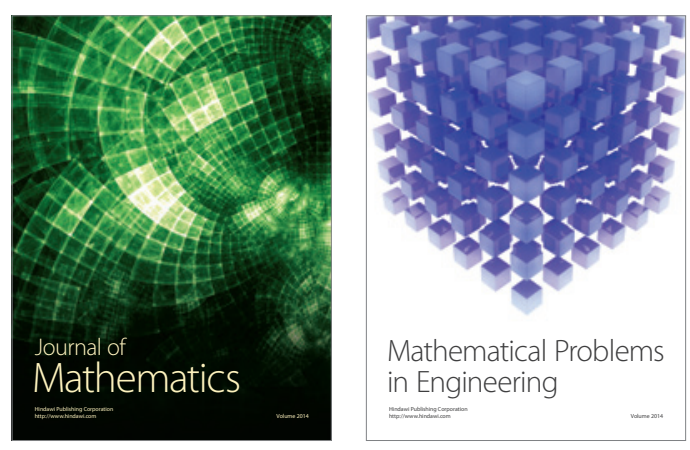

Mathematical Problems in Engineering
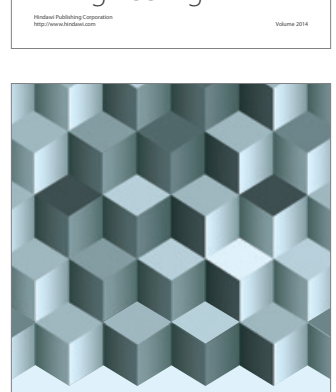

Journal of

Function Spaces
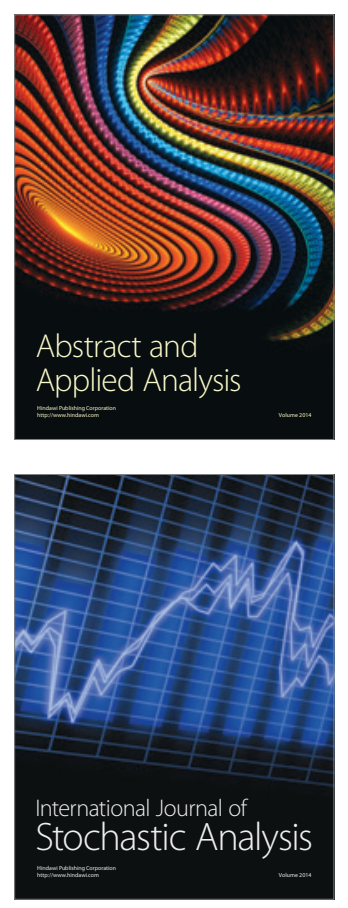

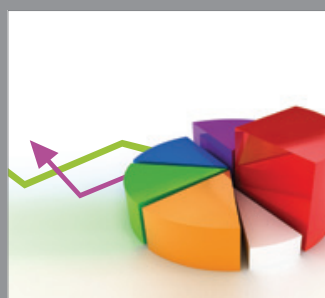

ournal of

Probability and Statistics

Promensencen
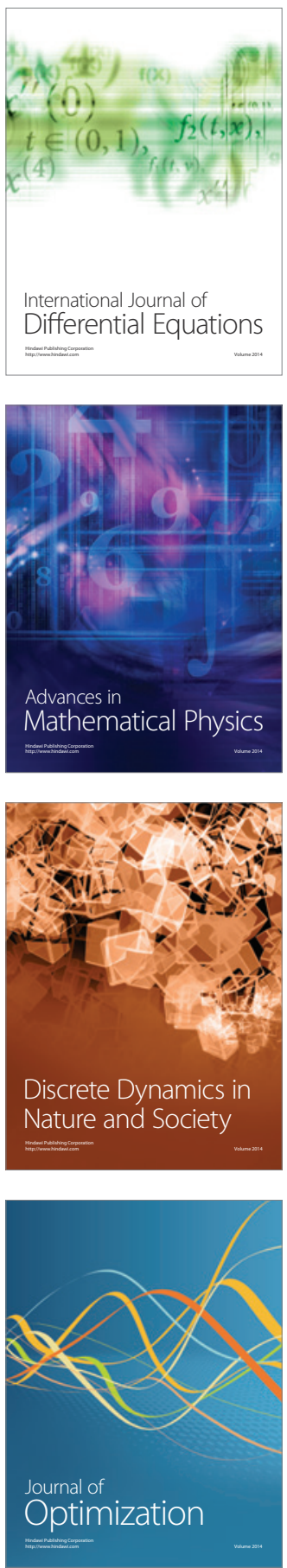